\title{
To court or not to court - a multimodal sensory decision in Drosophila males
}

\author{
Annika Rings* and Stephen F. Goodwin
}

\begin{abstract}
When Drosophila males encounter another fly, they have to make a rapid assessment to ensure the appropriate response: should they court, fight or pursue a different action entirely? Previous work has focused on the significance of sensory cues detected by the male during these encounters, however recent evidence highlights the importance of the male's own internal state in shaping his responses. Additionally, once triggered, courtship is not a rigid sequence of motor actions, but rather a finely-tuned behavioural display that must continually update in response to sensory feedback. Here, we review recent findings highlighting how sensory information and internal states are integrated ensuring appropriate action selection, and how they sustain and fine-tune motor output. We further discuss recent advances in our understanding of species differences in sensory processing that may contribute to reproductive isolation.
\end{abstract}

\section{Address}

Centre for Neural Circuits and Behaviour, University of Oxford, Tinsley Building, Mansfield road, Oxford, OX1 3SR, UK

*Corresponding author: Rings, Annika (annika.rings@dpag.ox.ac.uk) 


\section{Highlights}

- Multimodal sensory signals inform a male fly's decision to court.

- Internal states such as sleep drive and mating drive can bias this decision.

- The pC1 cluster integrates sensory input and internal states for action selection.

- Fine-tuning of specific courtship motor programs requires sensory feedback. 


\section{Introduction}

Innate sex-specific behaviours in Drosophila melanogaster have been exploited as a powerful model system to study how the brain evaluates sensory inputs and turns them into behavioural outputs. Two key transcription factors, doublesex $(d s x)$ and fruitless (fru), control the development of a sexually dimorphic nervous system, which underlies sexspecific behaviours, such as male courtship and aggression as well as female receptivity and postmating behaviours (reviewed in [1]). The details of female behaviour have been reviewed recently [2] and are beyond the scope of this review. Since sex-specific behaviours are hardwired into the fly nervous system via the actions of $d s x$ and fru, the focus has been on neurons that express these transcription factors to find anatomical or molecular sex differences in neuronal populations, and to gain entry to the neural circuits underlying sex-specific behaviours. Previous studies have identified the male-specific fru/dsx coexpressing $\mathrm{P} 1$ cluster, a subset of the $d s x$ expressing $\left(d s x^{+}\right) \mathrm{pCl}$ cluster, as a centre for courtship initiation [3]. This cluster, though not strictly necessary for courtship, can trigger wing extension when activated artificially or by female pheromones [3-5]. Here, we review recent evidence that P1 neurons integrate not only sensory information, but also internal states. We summarize recent work suggesting that P1 is involved in the choice between mutually exclusive behaviours, such as courtship, aggression and sleep, and therefore serves as a centre for action selection in male flies. We further present recent findings in other species which indicate that small changes in this conserved circuit can lead to reproductive isolation. Finally, we discuss how sensory feedback modulates courtship motor programs.

\section{Sensory processing}

Before a male invests time and energy into the pursuit of a potential mate, it is crucial that he accurately evaluates their suitability. To increase his chances of a successful mating and thus the production of offspring, the most relevant information he must gain during his encounter with another fly is its sex, species and mating status. In D. melanogaster, these traits are conveyed mainly by olfactory and gustatory pheromone signals, which have been recently reviewed in detail elsewhere [6]. Low volatility hydrocarbons such as 7,11-heptacosadiene $(7,11-H D)$ on the female cuticle promote 
courtship and are perceived by the male during the courtship ritual through tapping the female with his foreleg or licking [7]. On the other hand, malespecific gustatory and olfactory pheromones, such as 7-tricosene (7-T) and 11-cis-vaccenyl-acetate (cVA), inhibit male-male courtship and promote aggression [8,9]. Males also actively deposit cVA, creating 'landmarks' which are attractive to both sexes [10]. Notably, male pheromones are also transferred to females during copulation and thereby render mated females less attractive [11]. This preference for virgin females, while partly innate, also has a learned component, known as courtship conditioning $[12,13]$.

CRISPR/Cas9 technology has made it possible to investigate the underlying circuits in other Drosophila species that were previously genetically intractable $\left[14 * *, 15^{*}\right.$ ]. For example, recent work has found that pheromonedependent male preference, rather than female choice, is the main factor that blocks inter-species courtship in $D$. simulans $[14 * *, 16]$. In this species, females mainly produce 7-T and lack 7,11-HD, which is inhibitory to courtship in D. simulans males. Pheromone-perception circuits appear to be largely conserved, however, minor changes in the weight of excitation to inhibition onto P1 were identified as the potential basis for the switch from a stimulatory chemosensory signal in $D$. melanogaster to an inhibitory one in D. simulans [14**].

The role of acoustic signals in courtship has been extensively studied in females, as females evaluate male courtship song for species recognition and receptivity [17-20]. In males, the function of hearing in courtship is less clear. It has been suggested that males may use sound to localize females $[21,22]$, and that hearing courtship or aggression song can decrease or increase aggressiveness in males, respectively [23]. A recent study looking at both males and females found that previous exposure to their own species' song led to an increase in their preference for their species-specific song, suggesting a potential learned component to song discrimination [24]. The pathways underlying song perception in males, like females, have been shown to involve the $\mathrm{pCl}$ cluster [25], however, it is still unclear if this includes the P1 subset of $\mathrm{pCl}$.

The role of vision in courtship has recently received increased attention. Although vision alone can trigger short episodes of courtship, more persistent courtship requires additional inputs [26-28]. Vision mainly 
provides feedback for precisely controlled motor outputs, such as visual tracking [29*] and modulation of courtship song [30*,31]. A fru class of visual projection neurons, lobula columnar 10 (LC10), has recently been shown to regulate visual aspects of courtship behaviour [29*]. When LC10 neurons are artificially inactivated, males behave more like 'blind' flies, losing their ability to visually guide their behaviour towards the female, despite normal performance in other visual tests unrelated to courtship. Intriguingly, the anatomy and physiological responses of LC10 neurons are similar between the sexes, however, no clear behavioural function for LC10 neurons has been characterised in females [29*]. This suggests that males and females detect the same visual signals but process them differently to produce distinct behavioural responses. In a recent paper comparing gross $f r u^{+}$neural anatomy between $D$. melanogaster and $D$. subobscura (a species whose courtship relies heavily on visual cues [32]), the authors uncovered a conspicuous species-specific anatomical difference in the male optic lobe, suggesting a potential correlation between $\mathrm{fru}^{+}$visual pathway connectivity and the role of vision in courtship [15*,33].

\section{Internal states}

It has become increasingly evident that courtship is not merely a result of sensory input integration, but also depends on the internal state of the male. Although mating is a crucial behaviour for the survival of a species, competing needs of the individual, such as sleep drive or the presence of a competitor, might make it favourable to prioritise different behaviours.

A male may have very similar encounters, with comparable sensory stimuli, yet his reaction may differ depending on his motivational state, which is referred to as mating drive. Male mating drive depends on sexual experience: after several successful matings, the male's probability of initiating courtship towards an equally desirable female decreases, a behaviour that coincides with, but does not depend on, the depletion of ejaculatory fluid [34]. Recent work has identified key neurons expressing the neuromodulator dopamine as the neural basis of mating drive [34-36]. Intriguingly, it appears that dopamine directly modulates P1 neurons [34,35*], suggesting that P1 neurons might integrate internal states with sensory signals. 
The competing drives of sleep and courtship have recently been explored: sleep deprivation was shown to reduce courtship [37*], conversely, a strong courtship drive was found to reduce sleep, even in sleep-deprived males $[38,39 *]$. The decision to prioritise sleep vs. courtship is likely governed by the competition between sleep and mating drives, and depends on a $\mathrm{fr}^{+}$ cluster of clock neurons called DN1 [37*] and a newly discovered set of fru/dsx octopaminergic neurons called MS1 [39*], both of which appear to be functionally connected to P1 [37*,39*]. Consistent with this interplay between drives, P1 neuronal activation can suppress sleep [40] and DN1 plays a role in the circadian rhythmicity of male sex drive $[41,42]$.

Similar competing drives have also been shown between aggression and courtship. Aggression arousal levels represent a long-lasting internal state which can bias the decision whether to fight or court. Like mating drive, aggression levels are partly innate [43], but also depend on experience in previous aggressive encounters [44]. Interestingly, this 'winner and loser' effect not only alters aggressive potential, but also effects courtship [45].

\section{Command circuitry for action selection}

Which centre in the brain is responsible for integrating sensory information with internal states and deciding which action to take?

In order to arrive at the correct decision, a centre for action selection should

(i) Process sensory inputs as well as information about internal states

(ii) Initiate or suppress motor programs depending on the outcome of the calculation

Most efforts to determine a 'decision making' centre for male courtship have focused on the $\mathrm{pC} 1$ cluster. Figure 1 schematically illustrates the signals that are integrated by $\mathrm{pC} 1$, and its fru ${ }^{+}$subset, $\mathrm{P} 1$. There is clear evidence that $\mathrm{P} 1$ can be activated through sensory information received by gustatory pheromones [4] in addition to integrating information from other sensory modalities [28,46] (figure $1 \mathrm{~A}$ ), however, how these signals are processed by P1 is still unclear. In a recent study, Zhang et al. propose a model in which courtship initiation can be explained nearly completely by probabilistic activation of P1 neurons through tapping [35*]. In this view, motivational states mainly act by balancing excitatory and inhibitory inputs onto P1 and thereby changing the probability of its activation by a tap. 
Intriguingly, most internal states that have been implicated in courtship, such as sleep [37*], mating drive [34,35*] and aggression levels [47], have also been linked to P1 neurons (figure 1B). Most conflicting behaviours are suppressed by the activation of $\mathrm{P} 1$, however, $\mathrm{P} 1$ has been shown to induce both courtship and aggression [47]. How can one cluster promote both courtship and aggression, when those two behaviours rarely occur at the same time and in the same context, and when most pheromones have opposite effects on these behaviours? It has been suggested that these mutually exclusive behaviours are triggered by different activation levels [47] or different subsets of $d s x^{+} \mathrm{pCl}$ neurons [48], some of which are $\mathrm{fru}^{-}$ and therefore distinct from P1. In this context, it is important to note that the $\mathrm{P} 1$ cluster itself also constitutes a heterogeneous population of neurons [49], and a plethora of intersectional genetic techniques have been employed, which label varying subpopulations (for a detailed review, see [50]). An alternative hypothesis has been suggested by Hoopfer in a recent review [51]: P1 could simply signal the presence of a female as a limited resource, as aggressive behaviour requires the presence not only of a conspecific competitor, but also of a limited resource to fight over. Another possibility is that the differential effect is mediated by downstream targets of P1. Consistent with this hypothesis, a recently identified $\mathrm{fru}^{+}$set of neurons, aSP2, receives convergent inputs from $\mathrm{P} 1$ and from octopaminergic neurons and seems to be involved in P1's aggressionpromoting effect [52*]. In the case of courtship, several neurons downstream of P1 have been proposed [53], some of which also receive additional modulatory inputs $[30 *, 54]$. It is therefore tempting to speculate that these downstream targets also shape the decision to initiate specific motor programs.

\section{Sensory modulation of courtship motor output}

Several neuronal clusters have been identified which trigger courtship motor programs, and most prominently courtship song (recently reviewed in [55]). Although a recent study suggests that some motor actions, namely licking, abdominal bending and leg lifting, occur in a stereotyped sequence and are controlled by a single descending neuron [56], the courtship ritual as a whole is not a strict motor sequence, but instead is modulated by sensory feedback. Males use visual cues to modulate courtship song amplitude [31] and to choose between different song modes [30*] 
dependent on the distance of the female. Moreover, the male's orientation and wing choice during song production depend on visual control [29*] and are optimized to lead to high song amplitudes at the female antennae [57]. Unsurprisingly, following behaviour also requires visual control [29*]. Furthermore, it has been shown that sustaining courtship after initiation requires several sensory inputs [26-28], as well as continued activity of P1 neurons [35*]. A dopamine-gated recurrent activation loop has been proposed as an underlying mechanism for sustaining P1 activation [35*].

\section{Conclusion}

The male's decision to court depends on sensory signals of different modalities as well as various internal states. Current evidence supports the hypothesis that most of this information is integrated in the male-specific P1 cluster, which acts as a decision-making centre for male-specific behaviours. Recent evidence suggests that this circuitry is largely conserved among Drosophila species. Once the decision is made, specific motor programs are initiated by downstream neurons. These motor programs are not rigid sequences, but are dynamically updated by sensory signals.

Future research aimed at uncovering the circuits underlying sensory feedback will profit from large-scale connectomics projects, such as electron microscopic imaging of the whole fly brain [58]. Single-cell RNA sequencing can be used to reveal cellular heterogeneity and accurately characterise cell markers that are unique to different cell types [59,60]. Future studies may employ these techniques to identify functional subtypes of the pC1 cluster. Finally, CRISPR-Cas gene editing is revolutionising genetic analysis in insects, and is starting to shed light on the evolution of sexually dimorphic circuits in related Drosophila species $\left[14^{* *}, 15^{\star}, 61\right]$.

\section{Acknowledgements}

We thank all scientists whose results are reviewed in this article. We apologize to all the investigators whose work could not be cited owing to strict space constraints. We are grateful to the Wellcome Trust and the BBSRC for funding. We thank the members of the Goodwin laboratory for helpful discussions.

\section{References}

Papers of particular interest, published within the period of review, have been highlighted as: 
* of special interest

** of outstanding interest

1. Auer TO, Benton R: Sexual circuitry in Drosophila. Curr Opin Neurobiol 2016, 38:18-26.

2. Aranha $M M$, Vasconcelos $M L$ : Deciphering Drosophila female innate behaviors. Curr Opin Neurobiol 2018, 52:139-148.

3. Kimura K, Hachiya T, Koganezawa M, Tazawa T, Yamamoto D: Fruitless and doublesex coordinate to generate male-specific neurons that can initiate courtship. Neuron 2008, 59:759-769.

4. Kohatsu S, Koganezawa M, Yamamoto D: Female contact activates malespecific interneurons that trigger stereotypic courtship behavior in Drosophila. Neuron 2011, 69:498-508.

5. Inagaki HK, Jung Y, Hoopfer ED, Wong AM, Mishra N, Lin JY, Tsien RY, Anderson DJ: Optogenetic control of Drosophila using a red-shifted channelrhodopsin reveals experience-dependent influences on courtship. Nat Methods 2014, 11:325-332.

6. Yew JY, Chung H: Drosophila as a holistic model for insect pheromone signaling and processing. Curr Opin Insect Sci 2017, 24:15-20.

7. Billeter JC, Atallah J, Krupp JJ, Millar JG, Levine JD: Specialized cells tag sexual and species identity in Drosophila melanogaster. Nature 2009, 461:987-991. 
8. Wang L, Han X, Mehren J, Hiroi M, Billeter JC, Miyamoto T, Amrein H, Levine JD, Anderson DJ: Hierarchical chemosensory regulation of male-male social interactions in Drosophila. Nat Neurosci 2011, 14:757-762.

9. Kurtovic A, Widmer A, Dickson BJ: A single class of olfactory neurons mediates behavioural responses to a Drosophila sex pheromone. Nature 2007, 446:542-546.

10. Mercier D, Tsuchimoto Y, Ohta K, Kazama H: Olfactory Landmark-Based Communication in Interacting Drosophila. Curr Biol 2018, 28:2624-2631 e2625.

11. Everaerts C, Farine JP, Cobb M, Ferveur JF: Drosophila cuticular hydrocarbons revisited: mating status alters cuticular profiles. PLoS One 2010, 5:e9607.

12. Keleman K, Vrontou E, Kruttner S, Yu JY, Kurtovic-Kozaric A, Dickson BJ: Dopamine neurons modulate pheromone responses in Drosophila courtship learning. Nature 2012, 489:145-149.

13. Zhao X, Lenek D, Dag U, Dickson BJ, Keleman K: Persistent activity in a recurrent circuit underlies courtship memory in Drosophila. Elife 2018, 7.

**14. Seeholzer LF, Seppo M, Stern DL, Ruta V: Evolution of a central neural circuit underlies Drosophila mate preferences. Nature 2018, 559:564-569.

In this study, phemomone-perception circuits underlying courtship behaviour are investigated in $D$. simulans. A change in balance of excitation versus inhibition onto $\mathrm{P} 1$ neurons turns the $D$. melanogaster excitatory pheromone 
7,11 heptacosadiene into an inhibitory signal in $D$. simulans, thereby establishing a reproductive barrier between these species.

*15. Tanaka R, Higuchi T, Kohatsu S, Sato K, Yamamoto D: Optogenetic Activation of the fruitless-Labeled Circuitry in Drosophila subobscura Males Induces Mating Motor Acts. J Neurosci 2017, 37:11662-11674.

This paper analyses the frut circuitry in $D$. subobscura. The circuit is largely conserved, with some species-specific differences mainly in the visual system, and elicits subobscura-specific courtship behaviour when optogenetically activated.

16. Shahandeh MP, Pischedda A, Turner TL: Male mate choice via cuticular hydrocarbon pheromones drives reproductive isolation between Drosophila species. Evolution 2018, 72:123-135.

17. Clemens J, Ozeri-Engelhard N, Murthy M: Fast intensity adaptation enhances the encoding of sound in Drosophila. Nat Commun 2018, 9:134.

18. Vaughan AG, Zhou C, Manoli DS, Baker BS: Neural pathways for the detection and discrimination of conspecific song in D. melanogaster. Curr Biol 2014, 24:1039-1049.

19. Yamada D, Ishimoto $H$, Li X, Kohashi $T$, Ishikawa $Y$, Kamikouchi $A$ : GABAergic Local Interneurons Shape Female Fruit Fly Response to Mating Songs. J Neurosci 2018, 38:4329-4347. 
20. Zhou C, Pan Y, Robinett CC, Meissner GW, Baker BS: Central brain neurons expressing doublesex regulate female receptivity in Drosophila. Neuron 2014, 83:149-163.

21. Batchelor AV, Wilson RI: Sound localization behavior in Drosophila melanogaster depends on inter-antenna vibration amplitude comparisons. J Exp Biol 2019, 222.

22. Ejima A, Griffith LC: Courtship initiation is stimulated by acoustic signals in Drosophila melanogaster. PLoS One 2008, 3:e3246.

23. Versteven M, Vanden Broeck L, Geurten B, Zwarts L, Decraecker L, Beelen M, Gopfert MC, Heinrich R, Callaerts P: Hearing regulates Drosophila aggression. Proc Natl Acad Sci U S A 2017, 114:1958-1963.

24. Li X, Ishimoto $H$, Kamikouchi A: Auditory experience controls the maturation of song discrimination and sexual response in Drosophila. Elife 2018, 7.

25. Zhou C, Franconville R, Vaughan AG, Robinett CC, Jayaraman V, Baker BS: Central neural circuitry mediating courtship song perception in male Drosophila. Elife 2015, 4.

26. Agrawal S, Safarik S, Dickinson M: The relative roles of vision and chemosensation in mate recognition of Drosophila melanogaster. $J$ Exp Biol 2014, 217:2796-2805.

27. Krstic D, Boll W, Noll M: Sensory integration regulating male courtship behavior in Drosophila. PLoS One 2009, 4:e4457. 
28. Kohatsu S, Yamamoto D: Visually induced initiation of Drosophila innate courtship-like following pursuit is mediated by central excitatory state. Nat Commun 2015, 6:6457.

*29. Ribeiro IMA, Drews M, Bahl A, Machacek C, Borst A, Dickson BJ: Visual Projection Neurons Mediating Directed Courtship in Drosophila. Cell 2018, 174:607-621 e618.

Ribeiro et al. investigate the properties of LC10 visual projection neurons. They are shown to be important for visual tracking during courtship, and their physiological properties are tuned to the identification of fly-sized moving objects against a variable backgorund.

*30. Clemens J, Coen P, Roemschied FA, Pereira TD, Mazumder D, Aldarondo DE, Pacheco DA, Murthy M: Discovery of a New Song Mode in Drosophila Reveals Hidden Structure in the Sensory and Neural Drivers of Behavior. Curr Biol 2018, 28:2400-2412 e2406.

Using unsupervised machine based classification of Drosophila courtship song, the authors identify a new song mode by showing that pulse song can be subdivided into two distinct types. They further demonstrate that male song mode choice is affected by visual feedback.

31. Coen P, Xie M, Clemens J, Murthy M: Sensorimotor Transformations Underlying Variability in Song Intensity during Drosophila Courtship. Neuron 2016, 89:629-644.

32. Philip U, Rendel JM, Spurway H, Haldane JBS: Genetics and Karyology of Drosophila Subobscura. Nature 1944, 154:260. 
33. Higuchi T, Kohatsu S, Yamamoto D: Quantitative analysis of visually induced courtship elements in Drosophila subobscura. J Neurogenet 2017, 31:49-57.

34. Zhang SX, Rogulja D, Crickmore MA: Dopaminergic Circuitry Underlying Mating Drive. Neuron 2016, 91:168-181.

*35. Zhang SX, Miner LE, Boutros CL, Rogulja D, Crickmore MA: Motivation, Perception, and Chance Converge to Make a Binary Decision. Neuron 2018, 99:376-388 e376.

This study presents a coin-flip model of courtship initiation in response to tapping-induced activation of the P1 cluster. Dopaminergic inputs signalling mating drive and pheromonal inputs from unsuitable targets converge onto P1 neurons and modulate the probability of the binary decision. The paper further provides evidence for a recurrent activation loop that leads to P1 sustaining its own activation.

36. Lim J, Fernandez AI, Hinojos SJ, Aranda GP, James J, Seong CS, Han KA: The mushroom body D1 dopamine receptor controls innate courtship drive. Genes Brain Behav 2018, 17:158-167.

*37. Chen D, Sitaraman D, Chen N, Jin X, Han C, Chen J, Sun M, Baker BS, Nitabach MN, Pan Y: Genetic and neuronal mechanisms governing the sex-specific interaction between sleep and sexual behaviors in Drosophila. Nat Commun 2017, 8:154. 
This paper examines the relationship between sleep and courtship. Strong sleep drive can inhibit courtship, and the effect is mediated by $\mathrm{fru}^{+}$clock neurons called DN1, which are shown to be functionally connected to P1.

38. Beckwith EJ, Geissmann Q, French AS, Gilestro GF: Regulation of sleep homeostasis by sexual arousal. Elife 2017, 6.

*39. Machado DR, Afonso DJ, Kenny AR, Oztu Rk-Colak A, Moscato EH, Mainwaring B, Kayser M, Koh K: Identification of octopaminergic neurons that modulate sleep suppression by male sex drive. Elife 2017,6 .

This study identifies octopaminergic MS1 neurons that regulate sleep in males. These neurons are necessary for male sleep suppression in the presence of females and appear to be connected to fru ${ }^{+}$neurons.

40. Zhang W, Guo C, Chen D, Peng Q, Pan Y: Hierarchical Control of Drosophila Sleep, Courtship, and Feeding Behaviors by Male-Specific P1 Neurons. Neurosci Bull 2018, 34:1105-1110.

41. Fujii $S$, Amrein $H$ : Ventral lateral and DN1 clock neurons mediate distinct properties of male sex drive rhythm in Drosophila. Proc Natl Acad Sci U S A 2010, 107:10590-10595.

42. Fujii S, Emery $P$, Amrein H: SIK3-HDAC4 signaling regulates Drosophila circadian male sex drive rhythm via modulating the DN1 clock neurons. Proc Natl Acad Sci U S A 2017, 114:E6669-E6677.

43. Chowdhury B, Chan YB, Kravitz EA: Putative transmembrane transporter modulates higher-level aggression in Drosophila. Proc Natl Acad Sci U S A 2017, 114:2373-2378. 
44. Trannoy S, Penn J, Lucey K, Popovic D, Kravitz EA: Short and longlasting behavioral consequences of agonistic encounters between male Drosophila melanogaster. Proc Natl Acad Sci U S A 2016, 113:4818-4823.

45. Kim YK, Saver M, Simon J, Kent CF, Shao L, Eddison M, Agrawal P, Texada M, Truman JW, Heberlein U: Repetitive aggressive encounters generate a long-lasting internal state in Drosophila melanogaster males. Proc Natl Acad Sci U S A 2018, 115:1099-1104.

46. Clowney EJ, Iguchi S, Bussell JJ, Scheer E, Ruta V: Multimodal Chemosensory Circuits Controlling Male Courtship in Drosophila. Neuron 2015, 87:1036-1049.

47. Hoopfer ED, Jung Y, Inagaki HK, Rubin GM, Anderson DJ: P1 interneurons promote a persistent internal state that enhances inter-male aggression in Drosophila. Elife 2015, 4.

48. Koganezawa M, Kimura K, Yamamoto D: The Neural Circuitry that Functions as a Switch for Courtship versus Aggression in Drosophila Males. Curr Biol 2016, 26:1395-1403.

49. Costa M, Manton JD, Ostrovsky AD, Prohaska S, Jefferis GS: NBLAST: Rapid, Sensitive Comparison of Neuronal Structure and Construction of Neuron Family Databases. Neuron 2016, 91:293-311.

50. Asahina K: Sex differences in Drosophila behavior: Qualitative and Quantitative Dimorphism. Curr Opin Physiol 2018, 6:35-45. 
51. Hoopfer ED: Neural control of aggression in Drosophila. Curr Opin Neurobiol 2016, 38:109-118.

*52. Watanabe K, Chiu H, Pfeiffer BD, Wong AM, Hoopfer ED, Rubin GM, Anderson DJ: A Circuit Node that Integrates Convergent Input from Neuromodulatory and Social Behavior-Promoting Neurons to Control Aggression in Drosophila. Neuron 2017, 95:1112-1128 e1117.

This study identifies $f u^{+}$aSP2 neurons as an integration node for aggression, which receives convergent inputs from $\mathrm{P} 1$ and octopaminergic neurons and can bias behaviour to favor aggression over courtship.

53. von Philipsborn AC, Liu T, Yu JY, Masser C, Bidaye SS, Dickson BJ: Neuronal control of Drosophila courtship song. Neuron 2011, 69:509-522.

54. O'Sullivan A, Lindsay T, Prudnikova A, Erdi B, Dickinson M, von Philipsborn AC: Multifunctional Wing Motor Control of Song and Flight. Curr Biol 2018, 28:2705-2717 e2704.

55. Coen $P$, Murthy $M$ : Singing on the fly: sensorimotor integration and acoustic communication in Drosophila. Curr Opin Neurobiol 2016, 38:3845 .

56. McKellar CE, Lillvis JL, Bath DE, Fitzgerald JE, Cannon JG, Simpson JH, Dickson BJ: Threshold-Based Ordering of Sequential Actions during Drosophila Courtship. Curr Biol 2019, 29:426-434 e426. 
57. Morley EL, Jonsson T, Robert D: Auditory sensitivity, spatial dynamics, and amplitude of courtship song in Drosophila melanogaster. J Acoust Soc Am 2018, 144:734.

58. Zheng Z, Lauritzen JS, Perlman E, Robinson CG, Nichols M, Milkie D, Torrens O, Price J, Fisher CB, Sharifi N, et al.: A Complete Electron Microscopy Volume of the Brain of Adult Drosophila melanogaster. Cell 2018, 174:730-743 e722.

59. Croset V, Treiber CD, Waddell S: Cellular diversity in the Drosophila midbrain revealed by single-cell transcriptomics. Elife 2018, 7.

60. Li H, Horns F, Wu B, Xie Q, Li J, Li T, Luginbuhl DJ, Quake SR, Luo L: Classifying Drosophila Olfactory Projection Neuron Subtypes by SingleCell RNA Sequencing. Cell 2017, 171:1206-1220 e1222.

61. Ding Y, Lillvis JL, Cande J, Berman GJ, Arthur BJ, Long X, Xu M, Dickson BJ, Stern DL: Neural Evolution of Context-Dependent Fly Song. Curr Biol 2019, 29:1089-1099 e1087.

Figure 1: Schematic illustration of inputs to the courtship command cluster $\mathrm{pC} 1 / \mathrm{P} 1$

A. Sensory inputs to $\mathrm{pC} 1$ and its $\mathrm{fru}^{+}$subset, P1. Excitatory connections are shown in green, inhibitory connections are depicted in red, neuromodulation is 
shown in yellow. Dashed grey lines indicate putative connections that have not yet been identified.

B. Pathways signalling internal states to $\mathrm{pC} 1 / \mathrm{P} 1$ and putative interactions with gustatory circuits. Colour coding as in A.

The illustrations are based on data from the references cited in the text. 
A

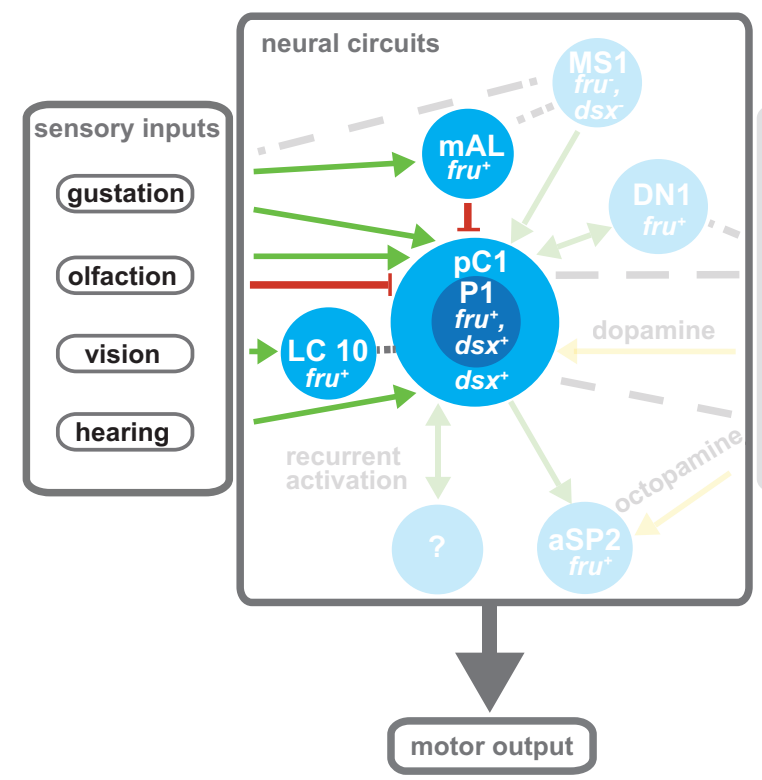

B

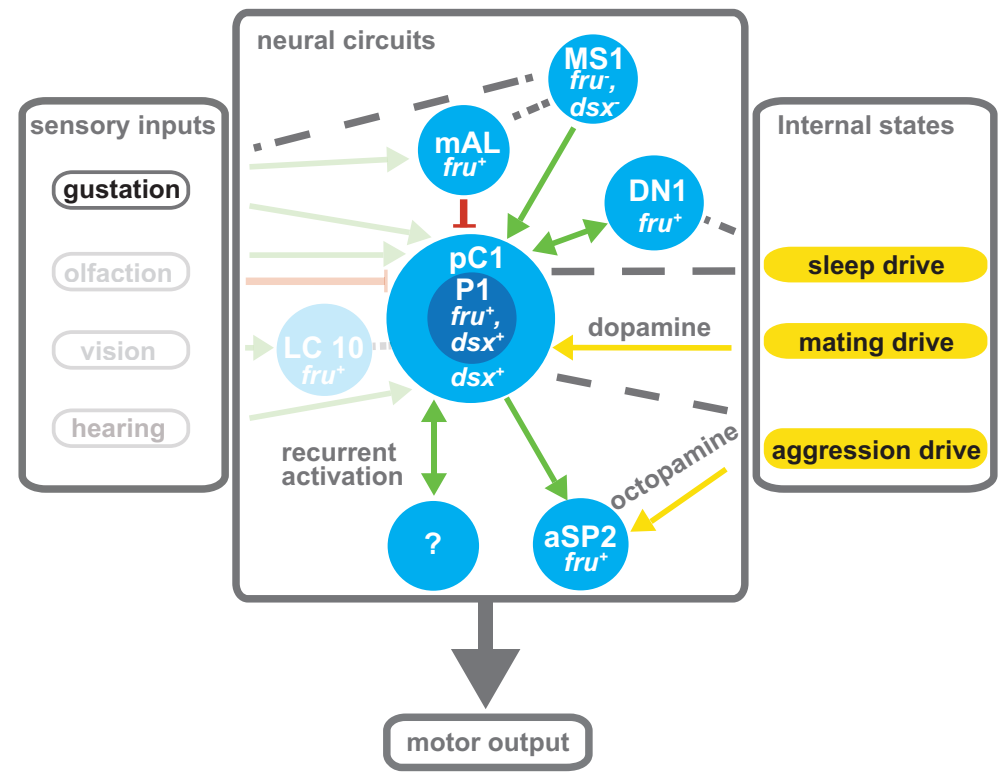

